\title{
A Fuzzy Interval Time Series Energy and Financial Forecasting Model Using Network-based Multiple Time-frequency Spaces and the Induced Ordered Weighted Averaging Aggregation Operation
}

\author{
Gang Liu, Fuyuan Xiao, Member, IEEE, Chin-Teng Lin, Fellow, IEEE, and Zehong Cao, Member, IEEE
}

\begin{abstract}
Forecasting time series is an emerging topic in operational research. Existing time series models have limited prediction accuracy when faced with the characteristics of nonlinearity and nonstationarity in complex situations related to energy and finance. To enhance overall prediction capabilities and improve forecasting accuracy, we propose a fuzzy interval time series forecasting model on the basis of network-based multiple time-frequency spaces and the induced ordered weighted averaging aggregation (IOWA) operation. Specifically, a time series signal is decomposed into ensemble empirical modes and then reconstructed as various time-frequency spaces, which are transformed into visibility graphs. Then, forecasting intervals in different spaces can be collected after the local random walker link prediction model is adopted. Furthermore, a rule-based representation value function inspired by Yager's golden rule approach is defined, and an appropriate representation value is calculated. Finally, after IOWA is used to aggregate the forecasting outcomes in different time-frequency spaces, the final forecast value can be obtained from the fuzzy forecasting interval. Considering that energy issues are of widespread interest in nature and the social economy, two cases, based on a hydrological time series from the Biliuhe River in China and two wellknown sets of financial time series data, TAIEX and HSI, are studied to test the performance of the proposed approach in comparison with existing models. Our results show that the proposed approach can achieve better performance than well-developed models.
\end{abstract}

Index Terms-Time series forecasting, financial time series, hydrological time series, ensemble empirical mode decomposition, network analysis, golden rule, link prediction, IOWA.

Corresponding author: Fuyuan Xiao.

Gang Liu and Zehong Cao contributed to this paper equally.

Gang Liu and Fuyuan Xiao are with the School of Computer and Information Science, Southwest University, Chongqing, 400715, China. (E-mail: xiaofuyuan@swu.edu.cn; doctorxiaofy@hotmail.com)

Chin-Teng Lin is with the Center for Artificial Intelligence, Faculty of Engineering and IT, University of Technology Sydney, Sydney, NSW, Australia (Email: Chin-Teng.Lin@uts.edu.au)

Zehong Cao is with The Discipline of ICT, School of Technology, Environments and Design, University of Tasmania, Hobart, TAS, Australia. (E-mail:zehong.cao@utas.edu.au)

\section{INTRODUCTION}

Energy plays a critical role in the modern economy and society. Many studies have focused on the estimation of energy demands, such as [1], [2]. Finance energy, an emerging subject that is receiving considerable attention, suggests viewing energy from a finance perspective. How to build a model with good performance [3], [4] and apply it to different applications [5]-[7], which relates to energy and finance issues, remains an open issue. Notably, many finance energy problems have inherent time-related characteristics, such as the periodic variations in wind speed and annual runoff. Normally, a time series is constituted by a sequence of related values ordered by time [8]-[10]. However, many realworld time series, such as stock indexes [11], [12] and annual runoff of rivers, have more uncertainty because of complex social and natural situations [13]. Because governments, environmentalists, investors, brokers and dealers all need to analyze the potential attributes in time series and predict future trends, many useful techniques and models have been proposed. Meanwhile, to achieve better performance, an increasing amount of research has recently begun to focus on improving the accuracy of forecasting [14].

Time series data can be divided into univariate and multivariate data. High-dimensional multivariate time series require the consideration of additional optimization algorithms [15], [16] for modeling and forecasting. However, these algorithms are not considered here since this paper focuses on univariate time series. There are many well-developed traditional time series forecasting methods, such as Box-Jenkins models, ranging from the autoregressive moving average (ARMA) to autoregressive integrated moving average (ARIMA) models [17], and fuzzy time series models, which are based on statistics or fuzzy logic and sets. However, the former requires the adoption of rather idealistic assumptions for simplicity and lack sufficient capacity to deal with nonlinear situations, whereas the latter requires cum- 
bersome calculations and involves equations that are difficult to understand. Due to these limitations, these methods are not suitable for application to real-world finance and energy time series. Recently, deep neural networks, such as long short-term memory (LSTM) networks and deep fuzzy neural networks (FNNs), have begun to be employed to achieve higher accuracy in time series forecasting [18], [19], but accurate rules still cannot be extracted due to black-box training processes and overfitting problems. To enhance the interpretability and predictability of neural networks, several approaches, such as intrinsic time-scale decomposition (ITD) [20], ensemble empirical mode decomposition (EEMD) [21], and fuzzy theories [22]-[25] have been proposed, which can perform well in specific forecasting situations. However, their adaptability to real-world applications is still restricted due to their poor shifting capability. Deep learning models also tend to have worse performance than traditional models due to overfitting issues. To mitigate these problems, it is necessary to collect a large amount of training data and use extensive computational resources; however, this is sometimes difficult to achieve in realistic situations.

In fact, most realistic time series are characterized by nonlinearity and nonstationarity, making it difficult for forecasting models to achieve high accuracy. Furthermore, driven by profits, benefits and other demands, investors or governments will never cease to pursue better performing models, especially in the context of energy or finance. Since many of the existing models listed above cannot perform well enough in complex, realistic situations, some researchers have recently investigated other possible solutions [26]-[30].

Recently, to study the potential of graph theory for time series analysis, Lacasa et al. [31] proposed the visibility algorithm, which can transform a time series into a complex network with well-conserved structural properties. Using this algorithm, many methods have been proposed to analyze time series from a network view [32], [33]. Among them, link prediction methods in networks [34], [35] are also considered to help construct the time series forecasting models. Many studies have begun to focus on this new perspective for time series forecasting and try to apply it in real energy and finance problems [36]. Compared to most traditional prediction models, the network approach requires less or even no parameters and shows no less performance. All of these methods show bright prospects for future network-based time series analysis.

Considering the current limitations imposed by the mercurial trends observed in time series from real-world applications and inspired by methods of fuzzy and interval time series analysis [37]-[39], we have explored the concept of information uncertainty and noted that a direct prediction approach may not be the best choice [40], [41]. Recent studies have presented various approaches for addressing different types of uncertainty [42]-[45]. For example, a fuzzy-based model is an efficient means of addressing linguistic variables and decision making [46]-[49], and interval values can be used to represent the properties of uncertain systems [50].

The forecasting model proposed in this study does not directly generate a deterministic predicted value; instead, it predicts an interval of values in the initial stage and then aggregates the outcomes. That is, in the prediction step, we first attempt to generate fuzzy predictions characterized by interval values, and we then apply a series of rules to choose a representative value to obtain the final prediction result. Furthermore, considering the achievements of network approaches for time series forecasting, we also attempt to investigate the time series from the network perspective to develop a more reliable forecasting model.

The model proposed in this paper first decomposes a time series into different intrinsic mode functions (IMFs) and a residue by means of the EEMD method. Then, these components are used to reconstruct a group of time series, which can be seen as representations of the original time series at different temporal scales or in different frequency states. After the reconstructed time series are generated, the visibility algorithm and the link prediction method can be adopted to produce interval forecasts for the different reconstructed time series. The final forecast result will be aggregated using the induced ordered weighted averaging aggregation (IOWA) operation [51], where the inducing variable is dynamically generated from the last forecast result in different time-frequency spaces. Throughout the whole process, the predictions are first considered as interval values, which can more properly represent uncertainty [52], [53]. Inspired by Yager's golden-rule-based representation values [54], the proposed model attempts to extract similar representation values according to the forecast context. The main contributions of our proposed model include 1) reconstructing a time series with different time-frequency components, 2) transforming these components into networks and then using link prediction to analyze them, 3) recovering interval time series from the analyzed networks, and 4) selecting representation values according to the forecast context and aggregating them to generate time series forecasts.

The remainder of this paper is organized as follows. Section II introduces some preliminaries about the visibility graph, the link prediction method, the IOWA operator and the reasoning algorithm with golden rules. Section III presents the proposed fuzzy interval value prediction model. In Section IV, application cases in real time series illustrate the performance of the proposed model. Section V finally presents a brief summary.

\section{Preliminaries}

In this section, some preliminaries about the visibility graph, the link prediction method, IOWA, fuzzy implication and the reasoning algorithm with golden rules are introduced sequentially. 

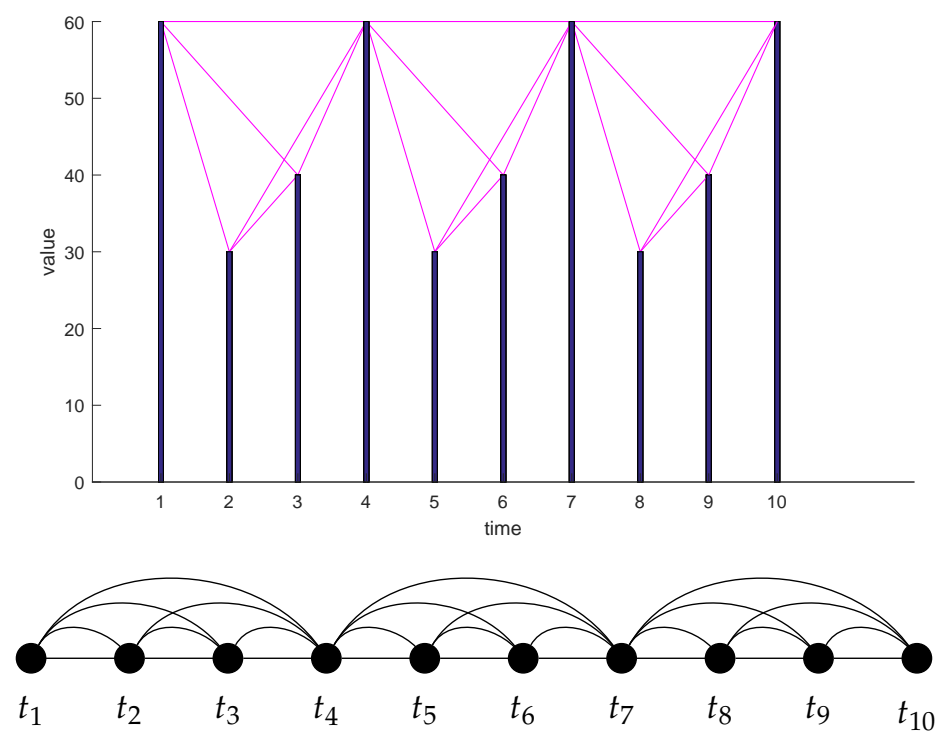

Fig. 1. The visibility algorithm is illustrated as follows. In the figure, if one bar can be seen from the top of another bar, then these two bars are linked, and the corresponding vertices in the associated visibility graph are also directly linked. For example, there is a visibility relationship between the two time series data $(1,60)$ and $(3,40)$ because there is a single time series data point $(2,30)$ between them and $30<(40+(60-40) \times(3-2) /(3-1))$. Therefore, there is a direct link between time nodes $t_{1}$ and $t_{3}$ in the visibility graph. By contrast, because there is a time series data point $(4,60)$ between $(3,40)$ and $(5,30)$ and $60>(30+(40-30) \times(5-4) /(5-3))$, the visibility criterion is not fulfilled for these points, meaning that there is no direct link between $t_{3}$ and $t_{5}$ in the visibility graph.

\section{A. The visibility graph}

The visibility graph [31] can serve as a bridge between time series and complex networks. Through the visibility algorithm, the structure of the time series can be conserved in the graph topology; for example, fractal time series become scale-free networks. In the visibility graph, two time series data $\left(t_{a}, y_{a}\right)$ and $\left(t_{b}, y_{b}\right)$ have a visibility relationship if there is no more than one time series data point $\left(t_{c}, y_{c}\right)$ between them and that point, if it exists, satisfies

$$
y_{c}<y_{b}+\left(y_{a}-y_{b}\right) \frac{t_{b}-t_{c}}{t_{b}-t_{a}} .
$$

An example of the visibility algorithm is presented in Fig. 1. Note that the visibility graph from the time series has the following properties:

1) Connected: each node sees at least its nearest neighbors.

2) Undirected: the links in the visibility graph have no direction.

3) Invariant under affine transformations of the series data: rescaling and translations of the horizontal and vertical axes do not influence the visibility criterion.

\section{B. Link prediction based on local random walk}

The real world is very complicated because various factors interact with each other in complicated ways [55]-[59]. Many mathematical models, such as DEMATEL [60]-[62] and Markov models [63], [64], have been presented to address this complexity. Among such models, the complex network is efficient for modeling the complexity by network analysis [65]. Link prediction is widely used in complex networks and attempts to explore the missing link in the network [66]. Most link prediction methods are based on node similarity. In the work [67], a method using the local random walk (LRW) approach is proposed, which measures the similarity between nodes by describing a walker walking randomly in a network [68]. Here, the term "random" implies that the method assumes that the future state of the walker is conditional on the current state and independent to the past, and the similarity between two nodes is defined as the likelihood of a link between them [67].

In this method, the footstep for a random walker can be represented by the probability transfer matrix $\boldsymbol{P}$. $\boldsymbol{P}_{x y}=a_{x y} / k_{x}$ means that the walker departs from one node $x$ and arrives at another node $y$ within one time step in a given network with $\mathrm{N}$ nodes. Note that $a_{x y}=1$ when two nodes have a link and $a_{x y}=0$ otherwise. $k_{x}$ is the degree of node $x$.

Initially, assume an $N \times 1$ vector, where $N$ is the length of the time series and is also the number of nodes in the network, with only the $x$-th element equal to 1 and the others equal to 0 ; this vector is denoted by $\vec{\pi}_{x}(0)$ and represents a random walker located at node $x$. After $t$ time steps, the vector $\vec{\pi}_{x}(t)$, representing the location probability for the random walker departing from $x$, can be calculated as follows:

$$
\vec{\pi}_{x}(t)=\boldsymbol{P}^{T} \vec{\pi}_{x}(t-1),
$$

where $T$ is the matrix transpose.

In accordance with the importance of each node in the graph, we can assign the initial resources for the 
LRW process [69]. For simplicity, here, we set the initial resources using the degrees of the nodes; then, the node similarity between $x$ and $y$ at time step $t$ can be defined as follows:

$$
S_{x y}^{L R W}(t)=\frac{k_{x}}{2|E|} \times \vec{\pi}_{x y}(t)+\frac{k_{y}}{2|E|} \times \vec{\pi}_{y x}(t),
$$

where $|E|$ is the number of edges in the network.

To prevent the difficulty that a sensitive dependence to parts of a network too far away from target nodes occurs in all random-walk-based similarity measures [70], the similarity index based on the superposed random walk (SRW) is used, as follows [67]:

$$
S_{x y}^{S R W}(t)=\sum_{n=1}^{t} S_{x y}^{L R W}(n)
$$

It is clear that $S_{x y}^{L R W}(n)=S_{y x}^{L R W}(n)$ and $S_{x y}^{S R W}(t)=$ $S_{y x}^{S R W}(t)$.

\section{Ensemble empirical mode decomposition}

The original empirical mode decomposition (EMD) method [71] is an adaptive and efficient approach for decomposing a time series into different IMFs and a residue. However, there is a problem called "modemixing", which means that any IMF is composed of oscillations of dramatically disparate scales in the original EMD method [21]. To overcome this shortcoming, the EEMD method is proposed as follows [21].

Assume that one original separately observed time series is $y(t)(t=1,2, \ldots, m)$. In EEMD, noise is introduced for every separate observation, and thus, the $i$-th "artificial" observation will be

$$
y_{i}(t)=y(t)+w_{i}(t),
$$

where $w_{i}(t)$ is regarded as the random noise that may be encountered in the measurement process. When there is only one observation, the multiple-observation ensembles can be mimicked by not arbitrary but rather varying realizations of added white noise, $w_{i}(t)$ [21]. [21]:

The basic procedure of EEMD is developed as follows

(1) add white noise to the observed time series.

(2) decompose the time series with white noise into IMFs.

(3) repeat steps (1) and (2) with different white noise series each time.

(4) obtain the final means of the corresponding IMFs and a residue.

The works [21], [71] illustrate that the IMFs must have the following properties or conditions:

1) The number of extrema and the number of zerocrossings must either be equal or differ by at most one through the whole size of an IMF.

2) The mean value of the upper envelope, which is defined by local maxima, and the envelope, which is defined by the local minima, is zero at any location.
According to the above definition, after a sifting process, any observed time series $y(t)(t=1,2, \ldots, m)$ can be decomposed to the following forms:

$$
y(t)=\sum_{i=1}^{n} c_{i}(t)+r_{n}(t),
$$

where $r_{n}(t)$ is the residue after $m$ IMFs are extracted. The sifting process is presented as follows:

(1) Identify all local maxima and minima for the observed time series $y(t)$;

(2) Form upper $e_{\max }(t)$ and lower $e_{\min }(t)$ envelopes by connecting all these local maxima and minima with cubic spline interpolation, and calculate the mean value, denoted by $e(t)$, between these two envelopes as follows:

$$
e(t)=\left(e_{\max }(t)+e_{\min }(t)\right) / 2 .
$$

(3) Extract $e(t)$ from the time series and obtain the first component $h(t)$ by taking the difference of $y(t)$ and $e(t)$ as follows:

$$
h(t)=y(t)-e(t) .
$$

(4) Check whether $h(t)$ satisfies the two conditions of IMF. If so, the first IMF, denoted by $c_{1}(t)$, equals $h(t)$. If not, $y(t)$ should be replaced by $h(t)$ and the above steps repeated until a $h(t)$ satisfies the two conditions of IMF. Then, the first residue $r_{1}(t)$ can be obtained as follows:

$$
r_{1}(t)=y(t)-c_{1}(t) .
$$

(5) Treat the residue as the new time series being subjected to the same sifting process shown above for the next IMF and residue as follows:

$$
\begin{gathered}
r_{2}(t)=r_{1}(t)-c_{2}(t), \\
r_{3}(t)=r_{2}(t)-c_{3}(t), \\
\cdots, \\
r_{n}(t)=r_{n-1}(t)-c_{n}(t) .
\end{gathered}
$$

(6) Finally, when the residue $r_{n}(t)$ becomes a monotonic function or the iteration number $n$ has met our conditions, the whole sifting procedure can be stopped [72].

\section{$D$. The induced ordered weighted averaging aggregation op- erator}

The ordered weighted averaging aggregation (OWA) and IOWA operations have both found widespread application [73]-[75] following their introduction by Yager [51], [76], [77]. They are both mappings, $F_{w}: R^{n} \rightarrow R$, and characterized by associated weighting vector $\vec{W}$. The main difference between OWA and IOWA is that the IOWA operator reorders the aggregated value $a_{i}$ with inducing variables.

In IOWA, the values waiting for aggregation are represented by tuple forms such as $\left\langle v_{i}, a_{i}\right\rangle$, where $v_{i}$ is the 
inducing variable. The entire aggregation process can be defined as follows:

$$
F_{\text {IOWA }}\left(\left\langle v_{1}, a_{1}\right\rangle, \ldots,\left\langle v_{i}, a_{i}\right\rangle\right)=\vec{W}^{T} \vec{B},
$$

where $\vec{W}$ and $\vec{B}$ are column vectors. $\vec{B}$ consists of the argument values $a_{i}$ ordered by the inducing variables $v_{i}$ in descending order.

Assuming that there are $n$ number values and that using $\alpha$ represents the degree to which this aggregation likes an or operation, the maximal entropy approach [78] can obtain the weights as follows:

1) If $n=2$, then $w_{1}=\alpha$ and $w_{2}=1-\alpha$.

2) If $\alpha=0$ or $\alpha=1$, then $w=(0,0, \cdots, 1)^{T}$ or $w=$ $(1,0, \cdots, 0)^{T}$, respectively.

3) If $n \geq 3$ and $0<\alpha<1$, then

$$
\begin{aligned}
& w_{1}\left[(n-1) \alpha+1-n w_{1}\right]^{n} \\
& =((n-1) \alpha)^{n-1}\left[((n-1) \alpha-n) w_{1}+1\right], \\
& w_{n}=\frac{((n-1) \alpha-n) w_{1}+1}{(n-1) \alpha+1-n w_{1}}, \\
& w_{j}=\sqrt[n-1]{w_{1}^{n-j} w_{n}^{j-1}} .
\end{aligned}
$$

\section{E. Reasoning algorithm and golden rules}

Uncertainty reasoning is a key issue in artificial intelligence. Many reasoning methods have been proposed. For example, based on evidence theory [79]-[82], evidential reasoning is widely used in decision making [83]-[85]. Compared with evidential reasoning, fuzzy set theory is used more extensively due to the relative simplicity of the corresponding reasoning process [59], [86]-[88]. To use a mathematical tool for modeling fuzzy systems in time series forecasting contexts, a reasoning algorithm based on fuzzy implication [89] is adopted. First, the format of fuzzy implication $R$ is suggested as follows:

$R$ : If $\left(x_{1}\right.$ is $A_{1}, \cdots, x_{k}$ is $\left.A_{k}\right)$, then $y=g\left(x_{1}, \cdots, x_{k}\right)$, where $x_{k}$ is the inferred value, $g(\cdot)$ is the function implying the value of $y$ under the premise, $x_{k}$ is the premise, and $A_{k}$ represents a fuzzy subspace where the implication $R$ can be applied for reasoning and is often a fuzzy set with a linear membership function.

Then, assume that there are $n$ fuzzy implications $R^{i}$ $(i=1, \cdots, n)$. For each implication $R^{i}$ :

1) There are $k$ premises $\left(x_{1}^{i}, \cdots, x_{k}^{i}\right)$ and fuzzy sets $\left(A_{1}^{i}, \cdots, A_{k}^{i}\right)$ and $y^{i}=g^{i}\left(x_{1}^{0}, \cdots, x_{k}^{0}\right)$.

2) Let $\left|y=y^{i}\right|$ represent the true value of the proposition $y=y^{i}$, which can be calculated as follows:

$$
\begin{aligned}
\left|y=y^{i}\right| & =\mid x_{1}^{0} \text { is } A_{1}^{i} \text { and } \cdots \text { and } x_{k}^{0} \text { is } A_{k}^{i}|\wedge| R^{i} \mid \\
& =\left(A_{1}^{i}\left(x_{1}^{0}\right) \wedge \cdots \wedge A_{k}^{i}\left(x_{k}^{0}\right)\right) \wedge\left|R^{i}\right| .
\end{aligned}
$$

For simplicity, assume that $\left|R^{i}\right|=1$.

After normalization, the final $y$ inferred from fuzzy implications is as follows:

$$
y=\frac{\sum\left|y=y^{i}\right| \times y^{i}}{\sum\left|y=y^{i}\right|}
$$

This is also called the Takagi-Sugeno approach [89].

Based on this approach, Yager et al. [54] proposed a golden-rule-based interval representation value for the unit interval. Assuming that there is an interval value of unit interval $[a, b]$, the representation value can be obtained by applying the function Rep as follows:

$$
\operatorname{Rep}(x)=m+\frac{1}{2} r-m r,
$$

where $m=(a+b) / 2$ and $r=b-a$. The corresponding golden rules are shown as follows [54]:

- If the mean is large and the range is small, then $\operatorname{Rep}(x)=1$;

- If the mean is large and the range is large, then $\operatorname{Rep}(x)=0.5$

- If the mean is small and the range is large, then $\operatorname{Rep}(x)=0.5$;

- If the mean is small and the range is small, then $\operatorname{Rep}(x)=0$.

\section{THE PROPOSED INTERVAL FORECASTING MODEL}

In this section, a new forecasting model is proposed. In general, a time series is first decomposed and reconstructed into multiple time-frequency spaces. Then, predictions for all subspaces are needed, and these results will be aggregated by the IOWA operator to make the eventual prediction. In this process, the prediction step is always divided into two parts. The first part is to generate a preliminary fuzzy interval value forecast, and the second is to select a representation value in this interval. Note that in this process, the upper and lower bounds of the interval value are real numbers; therefore, we need to extend the implementation of the golden-rule-based Takagi-Sugeno approach to include real numbers as well.

Note that the original golden rule method in the work [54] is not suitable for time series forecasting contexts, and to resolve this problem, we generalize the original golden rule method to select a proper 'golden representation value' from the forecasting interval.

\section{A. Decomposition and reconstruction}

The first step of the proposed method is to perform some time series pretreatments. This step mainly contains the decomposition and reconstruction parts. Assume that there is a time series $Y=$ $\left\{\left(t_{1}, y_{1}\right),\left(t_{2}, y_{2}\right), \ldots,\left(t_{N}, y_{N}\right)\right\}$. According to Equation (6), it can be decomposed into different IMFs and a residue $X=\left\{c_{1}, c_{2}, \cdots, c_{n} ; r_{n}\right\}$. Let $X(n+1)=r_{n}$; then, by reversing the order of $X$, a new sequence $X^{\prime}$ is obtained that satisfies

$$
X^{\prime}(j) \equiv X(i)
$$

where $i+j=n+2$. Therefore, the reconstructed time series in different time-frequency spaces, denoted by $\bar{X}_{i}$, can be obtained as follows:

$$
\bar{X}_{i}=\sum_{j=1}^{i} X^{\prime}(j) .
$$




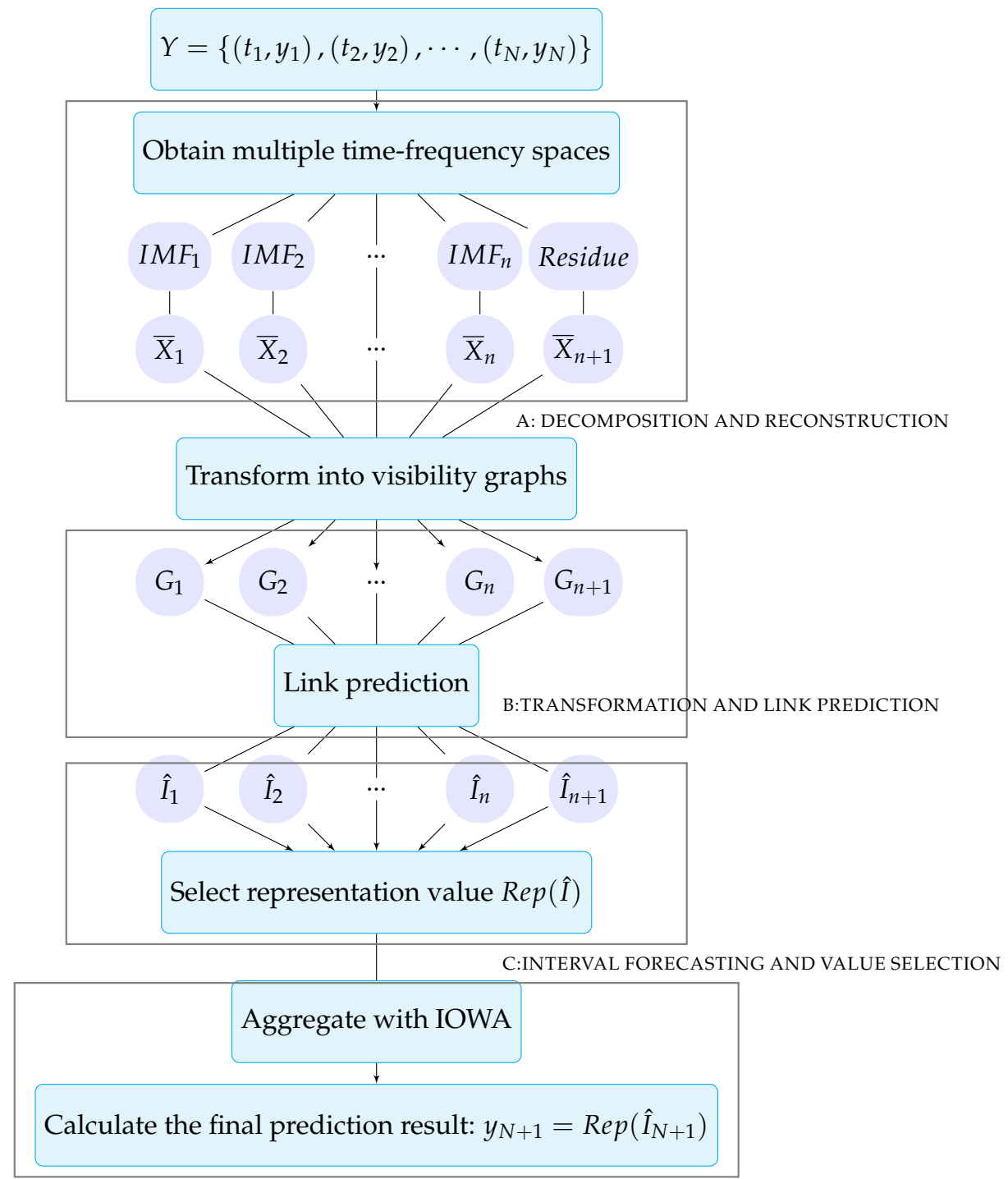

D: AGGREGATION AND FINAL PREDICTION

Fig. 2. The forecasting process of the proposed model

By gradually adding oscillations with different amplitudes and frequencies, it is clear that the original time series $Y$ can be reconstructed in $n+1$ time-frequency spaces and $\bar{X}_{n+1}=Y$.

\section{B. Transformation and link prediction}

For each reconstructed time series $\bar{X}_{i}(i=1,2, \cdots, n+$ 1 ), there is a corresponding visibility graph $G_{i}$ according to Equation (1). The link prediction method is then used to explore the most potential link between the future node and existing nodes in the current graph $G_{i}$. Because the future node does not exist, and inspired by past forecasting models such as exponential smoothing (ES), which express the idea that the current time is more important, let the most recent time node represent the next future node in the link prediction process [22].
Based on the LRW link prediction method, the transfer matrix $\boldsymbol{P}_{i}$ for each graph is first obtained. According to Equation (4), the similarity between the last node $N$ and the nodes in the preceding $(N-1)$ nodes can be calculated, and then, the similarity vector $\operatorname{Sim}_{i}$ for each graph $G_{i}$ is formed as follows:

$$
\operatorname{Sim}_{i}=\left[S_{i, 1 N}, S_{i, 2 N}, \ldots, S_{i, M N}, \ldots, S_{i,(N-1) N}\right] .
$$

Let $S_{i, M N}=\max \left(\operatorname{Sim}_{i}\right)$, that is, node $M$ is the most similar node to $N$ in $G_{i}$, and the corresponding time node in different time-frequency spaces is $\left(t_{M}, y_{M}^{i}\right)$. According to previous studies, the initial forecast value obtained by transforming a network into a time series satisfies [22], [90].

$$
y_{I n i}^{i}=\frac{y_{M}^{i}-y_{N}^{i}}{t_{M}-t_{N}}\left(t_{N+1}-t_{M}\right)+y_{M}^{i} .
$$




\section{Interval Forecasting and Value Selection}

Because Equation (12) is not a strict inversion of the visibility graph, it may lack a sufficient theoretical foundation. Here, we consider the preliminary prediction as intervals rather than exact values. This fuzzy method can intuitively help the forecast value reach the future real value. Thus, let $\hat{I}=\left[\kappa^{+}, \kappa^{-}\right]$denote the prediction interval. To forecast the value at $t_{N+1}$, these two boundaries can be obtained as follows:

$$
\begin{cases}\kappa_{i, N+1}^{+}=\max \left(\left\{y_{\text {Ini }}^{i}, y_{N}^{i}\right\}\right), & i=1,2, \cdots, n+1, \\ \kappa_{i, N+1}^{-}=\min \left(\left\{y_{\text {Ini }}^{i}, y_{N}^{i}\right\}\right), & i=1,2, \cdots, n+1\end{cases}
$$

Inspired by Yager's golden-rule-based representation values for the membership grades, the proposed model attempts to select a reasonable representation value for $\hat{I}$, denoted by $\operatorname{Rep}(\hat{I})$, in accordance with the following rules:

- If the forecast interval becomes smaller and the accuracy (error) becomes higher (smaller), then $\operatorname{Rep}(\hat{I})=\kappa^{+}$.

- If the forecast interval becomes larger and the accuracy (error) becomes higher (smaller), then $\operatorname{Rep}(\hat{I})=$ $\left(\kappa^{+}+\kappa^{-}\right) / 2=\bar{\kappa}$.

- If the forecast interval becomes smaller and the accuracy (error) becomes lower (larger), then $\operatorname{Rep}(\hat{I})=$ $\left(\kappa^{+}+\kappa^{-}\right) / 2=\bar{\kappa}$.

- If the forecast interval becomes larger and the accuracy (error) becomes lower (larger), then $\operatorname{Rep}(\hat{I})=$ $\kappa^{-}$.

Let $\delta$ denote the interval change rate, and let $\sigma$ denote the forecasting or estimated accuracy. Using the TakagiSugeno approach, this fuzzy system can be modeled as follows:

$$
\operatorname{Rep}(\hat{I})=\frac{\kappa^{+} \delta^{-} \sigma^{+}+\bar{\kappa} \delta^{+} \sigma^{+}+\bar{\kappa} \delta^{-} \sigma^{-}+\kappa^{-} \delta^{+} \sigma^{-}}{\delta^{-} \sigma^{+}+\delta^{+} \sigma^{+}+\delta^{-} \sigma^{-}+\delta^{+} \sigma^{-}},
$$

where $\delta^{+}\left(\delta^{-}\right)$represents the linguistic expression "the forecast interval becomes larger (smaller)", i.e., $A_{1}^{i}($ interval $)=\delta^{+}\left(\delta^{-}\right)$, and $\sigma^{+}\left(\sigma^{-}\right)$represents "the accuracy becomes higher (lower)", i.e., $A_{2}^{i}$ (accuracy) $=$ $\sigma^{+}\left(\sigma^{-}\right)$. Note that since we generalize the TakagiSugeno approach to the domain of real numbers, the min operation $\wedge$ in Equation (8) becomes a multiplication operation, and the true values of the fuzzy implications in these four golden rules should also be equal to 1 .

To further obtain the quantitative degree of semantic "forecast accuracy" and the "forecast interval", let $\|\hat{I}\|=$ $\kappa^{+}-\kappa^{-}$, and assume that $y_{N-1}$ is the real observed value for the last forecast time and that $\hat{y}_{N-1}$ is the last forecast result. Then, the interval change rate $\delta$ and the estimated accuracy $\sigma$ at time $N$ can be calculated as follows:

$$
\begin{gathered}
\delta=\frac{\left\|\hat{I}_{N}\right\|}{\left\|\hat{I}_{N-1}\right\|}, \\
\sigma=\max \left(\left\{\bar{\tau}-\frac{\left|\hat{y}_{N-1}-y_{N-1}\right|}{y_{N-1}}, \underline{\tau}\right\}\right),
\end{gathered}
$$

where $\bar{\tau}$ and $\underline{\tau}$ control the semantics of "a higher or lower accuracy degree". Moreover, $\bar{\tau}$ can be called the error tolerability parameter, and $\underline{\tau}$ should be an infinitesimal value. Higher tolerability means more uncertainty in the forecasting process. Theoretically, $\bar{\tau}$ can be any value, but for a realistic forecasting task, it is suggested that the range of $\bar{\tau}$ should be $[0.5,1.5]$, and in this paper, $\tau=1$ is adopted.

Note that in the original reasoning algorithm, $A$ is a fuzzy set with a linear membership function. However, in the proposed model, $A$ is not subject to these conditions, and it is decided by the last prediction result according to $\delta^{+}=\max (\{\delta, 1 / \delta\}), \delta^{-}=\min (\{\delta, 1 / \delta\})$ and $\sigma^{+}=\max (\{\sigma, 1 / \sigma\})$ and $\sigma^{-}=\min (\{\sigma, 1 / \sigma\})$. Thus, the golden rule representation value for the forecast interval can be rewritten as follows:

$$
\begin{aligned}
\operatorname{Rep}(\hat{I})= & \frac{\kappa^{+} \delta \sigma+\bar{\kappa}(1 / \delta) \sigma+\bar{\kappa} \delta(1 / \sigma)+\kappa^{-}(1 / \delta)(1 / \sigma)}{\delta \sigma+(1 / \delta) \sigma+\delta(1 / \sigma)+(1 / \delta)(1 / \sigma)} \\
= & \frac{\bar{\kappa}\left(\delta^{2}+\sigma^{2}\right)+\kappa^{+} \delta^{2} \sigma^{2}+\kappa^{-}}{\left(\delta^{2}+1\right)\left(\sigma^{2}+1\right)} .
\end{aligned}
$$

The initial values for $\delta$ and $\sigma$ are 1 . Therefore, for the first prediction result,

$$
\operatorname{Rep}(\hat{I})=\frac{2 \bar{\kappa}+\kappa^{+}+\kappa^{-}}{4}=\bar{\kappa} .
$$

Overall, the golden rule representative value is based on the Takagi-Sugeno (T-S) approach, which uses four rules (e.g., fuzzy implications) to control the representative value. For comparison, we use two variables as premises, as done in Yager's study [91] (the terms "mean" and "range" are used in Yager's study; the terms "forecast error" and "interval" are used in our study), and the inferred consequence ( $y$ ) can be obtained from the interval values' upper and lower bounds. However, since both the upper and lower bounds of the interval forecast are real numbers, the proposed model further improves the golden-rule-based representation value method of the T-S approach to adapt it to the time series context.

\section{Aggregation and final prediction}

After the forecasting results are obtained from every time-frequency time series, the IOWA method is adopted to aggregate the results to perform the final forecasting. Assume that a time series $Y=$ $\left\{\left(t_{1}, y_{1}\right),\left(t_{2}, y_{2}\right), \ldots,\left(t_{N}, y_{N}\right)\right\}$ has been decomposed and reconstructed to $\mathrm{n}+1$ time-frequency spaces $\bar{X}_{i}(i=$ $1,2, \cdots, n+1)$. At each time node $t_{N}$, after $n+1$ interval predictions from multiple time-frequency spaces are made, the representation values for each of them are also selected. Then, assume that they are $\operatorname{Rep}\left(\hat{I}_{1, N+1}\right), \operatorname{Rep}\left(\hat{I}_{2, N+1}\right), \cdots, \operatorname{Rep}\left(\hat{I}_{n+1, N+1}\right)$. Using the IOWA operator, the final forecasting interval satisfies

$$
\left\{\begin{array}{l}
\kappa_{N+1}^{+}=\max \left(\left\{R_{\text {IOWA }}, y_{N}\right\}\right), \\
\kappa_{N+1}^{-}=\min \left(\left\{R_{I O W A}, y_{N}\right\}\right),
\end{array}\right.
$$


where $i=1,2, \cdots, n+1$, and $R_{I O W A}$ is the aggregated result using the IOWA method as follows:

$$
R_{\text {IOWA }}=F_{\text {IOWA }}\left(\left\langle v_{1}, \operatorname{Rep}\left(\hat{I}_{1, N+1}\right)\right\rangle, \ldots,\left\langle v_{i}, \operatorname{Rep}\left(\hat{I}_{i, N+1}\right)\right\rangle\right) \text {. }
$$

where the inducing variables $v_{i}$ can be obtained as follows:

$$
v_{i}=\frac{1}{\sum_{k=1}^{N-1}\left|\hat{y}_{i, k}-y_{i, k}\right|} .
$$

Therefore, we have $\hat{I}_{N+1}=\left[\kappa_{N+1}^{-}, \kappa_{N+1}^{+}\right]$as the final interval forecast. Then, the golden representation value for this forecasting interval can be obtained as follows:

$$
\hat{y}_{N+1}=\operatorname{Rep}\left(\hat{I}_{N+1}\right) \text {. }
$$

The whole forecasting process is shown in Fig. 2.

\section{Application}

Energy issues involve every aspect of life, including stock markets and water resource management. In this section, two types of cases are considered to verify the performance of the proposed time series model, especially in energy-related forecasting. In the first case, a hydrological time series constructed by the annual runoff of the Biliuhe reservoir from Liaoning Province, China, is used. In the second case, two well-known financial time series, TAIEX and HSI, are used. In addition, some baseline or recent well-developed methods are also taken into account for comparison to illustrate the performance of the proposed model.

\section{A. Dataset introduction and performance criteria}

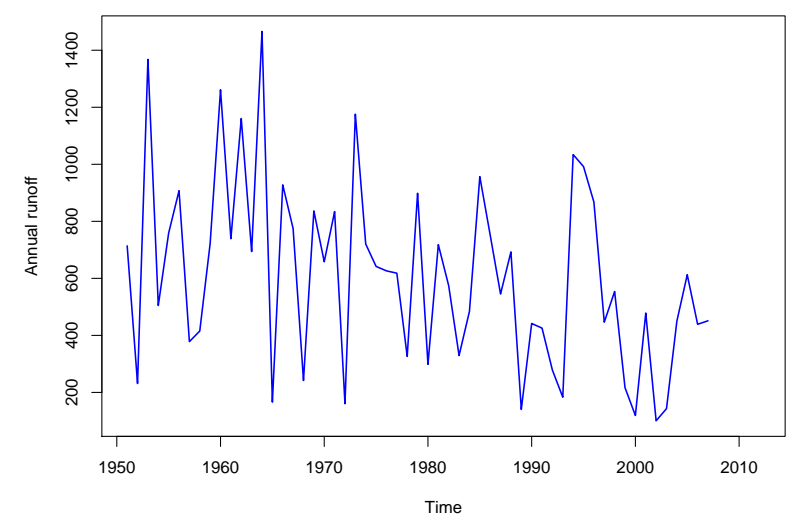

Fig. 3. Hydrological time series of Biliuhe Reservoir $\left(10^{6} \mathrm{~m}^{3}\right)$

The study area of Case 1 is the Biliuhe River, with an average annual rainfall of approximately $742.8 \mathrm{~mm}$ in the catchment. Forecasting the annual runoff of reservoirs such as the Biliuhe River is meaningful because it can contribute to the designs of many environmental projects near the river, help plan the plant irrigation system, and maintain the sustainable utilization of water resources and energy [14]. In this experiment, the Biliuhe annual runoff data from 1951 to 2007 are collected. Data from
1951 to 1995 are used as the training set, and data from 1996 to 2007 are used as the validation set. Fig. 3 shows the whole dataset.

Case 2 contains two experiments, both of which are financial time series for important stock markets. Energy is currently playing an increasingly important role in financial markets. Energy prices such as oil and sustainable energy cost are of great relevance to international markets. In addition, the emerging inherently interdisciplinary subject of energy finance also requires us to consider energy problems from a financial perspective. Therefore, in the second study case, the daily stock index of the Taiwan Stock Exchange Capitalization Weighted Stock Index (TAIEX), which covers the duration from 1998 to 2006, is first used. For most existing forecasting models, the training data are from January to October, while the prediction data are from November to December. However, in the proposed model, there is no need to keep a very large training set, and here, we let the size of the training set be only 10 , which is far less than that of the original.

The second experiment utilizes the Hang Seng Index (HSI) collected from 1998 to 2006. Similarly, the size of the training set is 10, and the data waiting to be predicted are from November to December every year.

Thus, we follow this experimental paradigm to provide an easy starting point for performance comparisons.

Note that here, we follow the experimental paradigm previously applied in references [92], [93] to make it easy to verify the feasibility of the proposed method and perform comparisons.

After the prediction results are obtained, many criteria can be selected to evaluate the proposed model. The first one is the root mean square error (RMSE), as follows:

$$
R M S E=\sqrt{\frac{1}{N} \sum_{i=1}^{N}\left(Q_{f}(i)-Q_{0}(i)\right)^{2}},
$$

where $Q_{f}(i)$ and $Q_{0}(i)$ are the forecast and real time series data, respectively.

The second is the mean absolute percentage error $(M A P E)$, as follows:

$$
M A P E=\frac{1}{N} \sum_{i=1}^{N}\left|\frac{Q_{f}(i)-Q_{0}(i)}{Q_{0}(i)}\right| \times 100 .
$$

The third is the correlation coefficient (Corr), as follows:

$$
\text { Corr }=\frac{(1 / N) \Sigma_{i=1}^{N}\left(Q_{0}(i)-\bar{Q}_{0}\right)\left(Q_{f}(i)-\bar{Q}_{f}\right)}{\sqrt{(1 / N) \sum_{i=1}^{N}\left(Q_{0}(i)-\bar{Q}_{0}\right)^{2}} \times \sqrt{(1 / N) \Sigma_{i=1}^{N}\left(Q_{f}(i)-\bar{Q}_{f}\right)^{2}}} .
$$

The fourth is the Nash-Sutcliffe efficiency coefficient (NSE) [94], as follows:

$$
N S E=1-\frac{\sum_{i=1}^{N}\left(Q_{0}(i)-Q_{f}(i)\right)^{2}}{\sum_{i=1}^{N}\left(Q_{0}(i)-\bar{Q}_{0}\right)^{2}} .
$$

Normally, RMSE is the most commonly used evaluation criterion, and the NSE coefficient, which ranges in 
value from $-\infty$ to 1 with a best score of 1 , is often used to assess the forecasting performance of hydrological models.

Note that both of the above experiments are of the onestep-ahead type, and the algorithm process is presented in Algorithm 1.

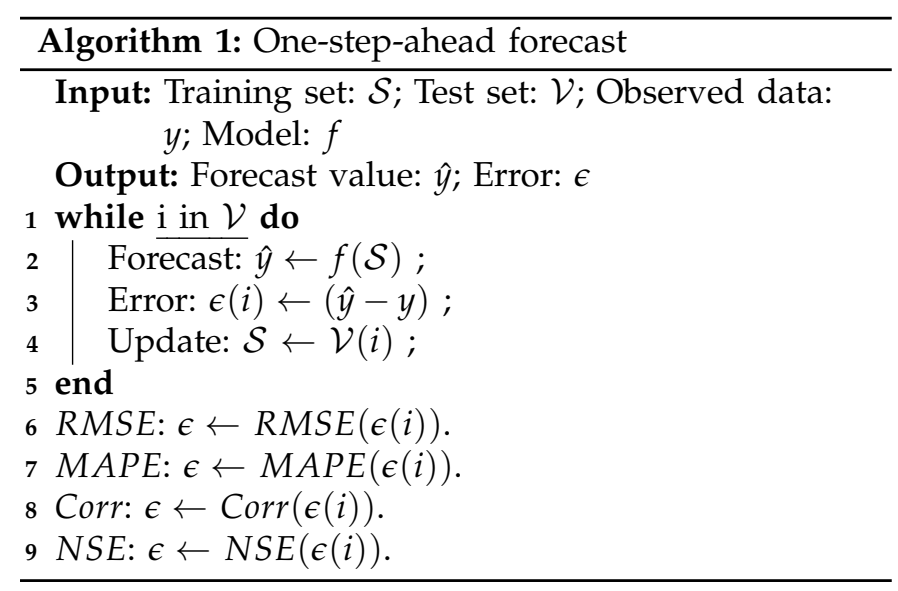

\section{B. Case study 1: Hydrological time series forecasting}

TABLE I

HYDROLOGICAL TIME SERIES FORECASTING RESULTS WITH $\alpha=0.8$ FROM 1996 TO $2007\left(10^{6} \mathrm{~m}^{3}\right)$.

\begin{tabular}{cccl}
\hline Year & Real value & The proposed model & Error \\
\hline 1996 & 867.48 & 924.1297 & 56.6497 \\
1997 & 446.37 & 659.3244 & 212.9544 \\
1998 & 553.82 & 475.2472 & 78.5728 \\
1999 & 216.61 & 526.6286 & 310.0186 \\
2000 & 119.18 & 235.4719 & 116.2919 \\
2001 & 478.32 & 215.3422 & 262.9778 \\
2002 & 100.52 & 415.2108 & 314.6908 \\
2003 & 143.08 & 150.1904 & 7.1104 \\
2004 & 449.83 & 301.4942 & 148.3358 \\
2005 & 612.73 & 456.1894 & 156.5406 \\
2006 & 438.99 & 535.2381 & 96.2481 \\
2007 & 451.19 & 467.1541 & 15.9641 \\
\hline
\end{tabular}

The Biliuhe Reservoir plays an important role in supplying municipal water for Dalian City, which is located in Liaoning Province, China. Thus, past studies have often focused on analyzing the reservoir's annual runoff data such that they can make a proper prediction for the future [95]. In this paper, to forecast the annual runoff level of the Biliuhe River from 1996 to 2007, the proposed model first makes interval value predictions in reconstructed multiple frequency spaces for the original hydrological time series. Then, by using the IOWA approach (with $\alpha=0.8$ ) and golden rule representation values, the final forecasting results are obtained, as shown in TABLE I.

Because we consider the forecasting problem only from a single-variable perspective, some results achieved by the proposed model, such as for the year 2001, appear to be poor. To further illustrate the performance of the proposed model, we introduce two baseline forecasting models as comparisons. The first is the seasonal autoregressive integrated moving average (SARIMA) model [96]. SARIMA means that the time series may contain seasonal elements and it is often denoted by $S A R I M A(p, d, q)(P, D, Q)_{m}$, in which $m$ refers the number of periods for a season, $p, d, q$ represent the order of autoregressive model, differencing degree, moving average model, respectively, and $P, D, Q$ represent the seasonal part of the corresponding model. The second is a typical three-layer feedforward artificial neural network (ANN). The only parameter $\alpha$ in the proposed model is the "orness" level in the maximum entropy IOWA approach, which is set as $0.7,0.8$ or 0.9 in each time forecasting. Then, the corresponding results are as shown in TABLE II. It is evident that the proposed model improves the accuracy compared to SARIMA or ANN. In addition, we also find that by setting different $\alpha$, the proposed model may have different performances. TABLE II also shows that the "orness" level in the aggregation step has only a limited influence on the final forecasting results.

TABLE II

THE FORECASTING RESULTS FROM 1996 TO 2007 COMPARED WITH BASELINE METHODS.

\begin{tabular}{llllll}
\hline Model & & RMSE & MAPE & Corr & NSE \\
\hline & $\alpha=0.7$ & 189.79 & 66.24 & 0.618 & 0.242 \\
The proposed model & $\alpha=0.8$ & 180.13 & 63.84 & 0.656 & 0.318 \\
& $\alpha=0.9$ & 174.31 & 63.15 & 0.679 & 0.360 \\
SARIMA $(3,1,1)(1,0,1)_{6}$ & & 286.64 & 122.94 & -0.105 & -0.730 \\
ANN & & 342.85 & 149.05 & 0.135 & -1.474 \\
\hline
\end{tabular}

\section{Case study 2: Financial time series forecasting}

In the first experiment of case study 2, the parameter $\alpha$ is first set as 0.8 . Fig. 4 presents the first experiment forecast results compared with the actual observed time series. We find that the proposed model is conservative when it makes a prediction for a maximum.

To further study the performance of the proposed model, some classic benchmarks are used to evaluate the potential of the proposed method. In addition, some recent alternative approaches, namely, the fuzzy time series with genetic algorithm (FTSGA) model [98] which is a hybrid model combing genetic algorithm and fuzzy time series, the multi-order (first-, second-, third-order) fuzzy time series model [92] combined with genetic algorithm and technical analysis such as the Rate of Change, the method of integrated nonlinear feature selection (INFS) coupled with supportive vector regression (SVR) [93], and INFS based on an adaptive neural fuzzy inference system (ANFIS) time series model [93], are selected for comparison. Note that in the latter two methods, the INFS method is usually used to choose important technical indicators, based on SVR or ANFIS [93]. The classic methods considered are the firstand second-order autoregression $(A R(1)$ and $A R(2))$ or 


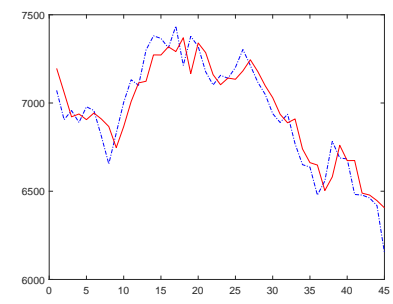

(a) 1998

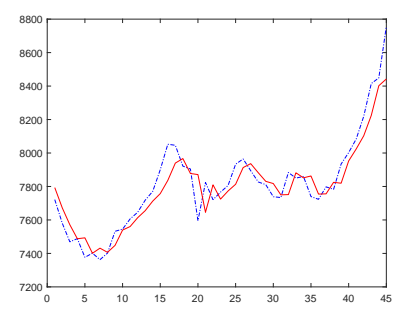

(b) 1999

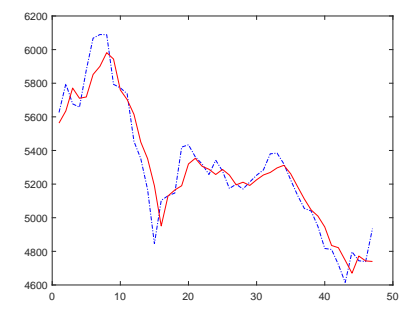

(c) 2000

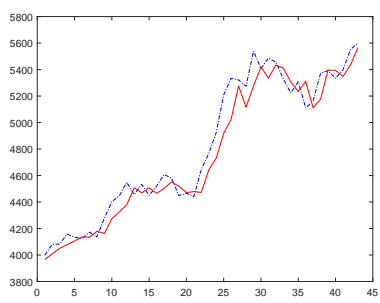

(d) 2001

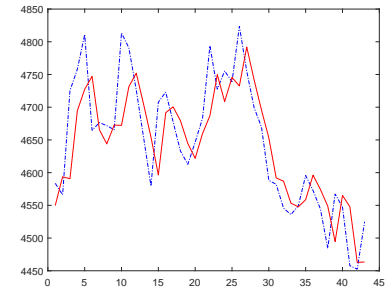

(e) 2002

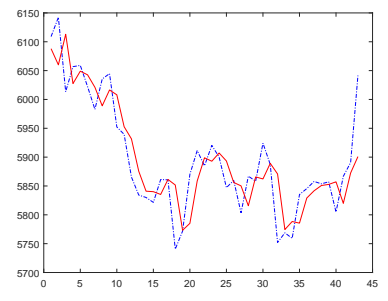

(f) 2003

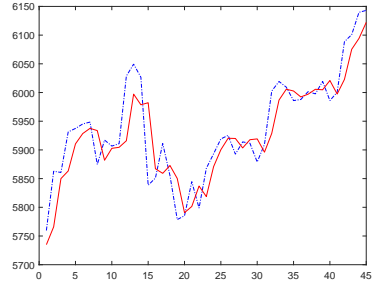

(g) 2004

Fig. 4. TAIEX forecasting results from 1997 to 2003

TABLE III

A PERFORMANCE COMPARISON (RMSE) FOR FORECASTING TAIEX FROM 1998 TO 2003.

\begin{tabular}{lllllllll}
\hline Models & 1998 & 1999 & 2000 & 2001 & 2002 & 2003 & 2004 & Average \\
\hline AR(1) [97] & 144.53 & 116.84 & 155.12 & 112.39 & 97.09 & 91.67 & 79.94 & 113.94 \\
AR(2) [97] & 135.21 & 128.15 & 142.30 & 129.84 & 89.80 & 66.58 & 60.33 & 107.46 \\
FTSGA [98] (2013) & 112.96 & 102.74 & 126.68 & 115.79 & 65.56 & 57.40 & 56.10 & 91.03 \\
INFS+SVR [93] & 117.28 & 118.91 & 148.52 & 110.76 & 70.38 & 59.22 & 53.07 & 96.88 \\
Multiorder fuzzy [92] (2016) & 114.21 & 110.09 & 125.42 & 113.22 & 63.99 & 52.99 & 52.40 & 90.33 \\
ANFIS + INFS [93] $(2016)$ & 121.18 & 112.11 & 132.19 & 113.23 & 65.83 & 57.62 & 54.33 & 93.78 \\
The proposed model $(\alpha=0.8)$ & 108.06 & 107.69 & 114.08 & 120.97 & 58.38 & 50.74 & 45.38 & 86.47 \\
\hline
\end{tabular}

TABLE IV

PERFORMANCE IMPROVEMENT DEGREE (\%) FOR FORECASTING TAIEX FROM 1998 TO 2003.

\begin{tabular}{lllllllll}
\hline Models & 1998 & 1999 & 2000 & 2001 & 2002 & 2003 & 2004 & Average \\
\hline AR(1) [97] & 25.23 & 7.83 & 26.46 & 7.63 & 39.87 & 44.65 & 43.23 & 24.11 \\
AR(2) [97] & 20.08 & 15.97 & 19.83 & 6.83 & 34.99 & 23.79 & 24.78 & 19.53 \\
FTSGA [98] (2013) & 4.34 & -4.82 & 9.95 & -4.47 & 10.95 & 11.60 & 19.11 & 5.01 \\
INFS+SVR [93] & 7.86 & 9.44 & 23.19 & -9.22 & 17.05 & 14.32 & 14.49 & 10.75 \\
Multiorder fuzzy [92] (2016) & 5.38 & 2.18 & 9.04 & -6.85 & 8.77 & 4.25 & 13.40 & 4.27 \\
ANFIS + INFS [93] (2016) & 10.83 & 3.94 & 13.70 & -6.84 & 11.32 & 11.94 & 16.47 & 7.79 \\
\hline
\end{tabular}

ARIMA models [97] and the choice depends on the autocorrelation function (ACF) and partial autocorrelation function (PACF) analysis. Statistical comparisons are shown in Table III. From this table, it is clear that the proposed model performs better in most situations. More improvement details are shown in TABLE IV, where we can find that except for the prediction result in 2001, the performance of the proposed model is comparatively stable, and on average, the model actually achieves improved forecasting accuracy (from $4 \%$ to $25 \%$ ).

Fig. 5 shows the result for the second experiment, and the prediction results are shown in TABLE V. Similar to the last experiment, the "orness" $\alpha=0.8$. In TABLE VI, it is evident that in most situations, the proposed model can improve the accuracy by $10 \%$ compared to recent time series forecasting models. In 2004, the forecast result of the proposed model is not as good as that of the multiorder fuzzy time method [92], but its accuracy is still improved by approximately $3.03 \%$ on average. Furthermore, the proposed model even achieves a $14.10 \%$ average improvement in forecasting accuracy compared with the INFS and SVR hybrid methods and an average improvement of approximately $8 \%$ compared with the 


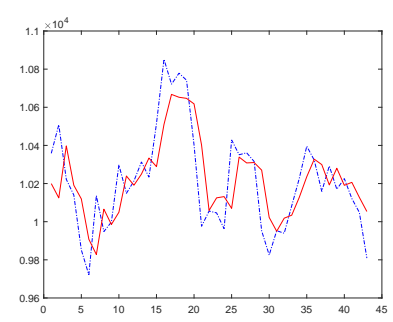

(a) 1998

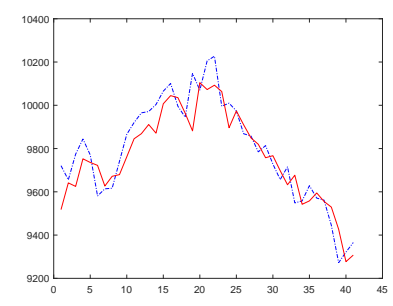

(e) 2002

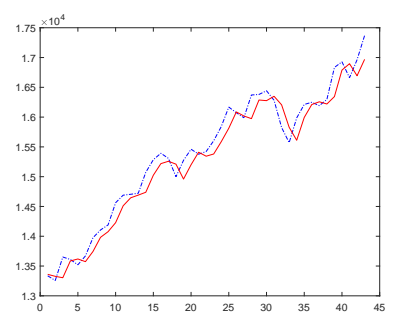

(b) 1999

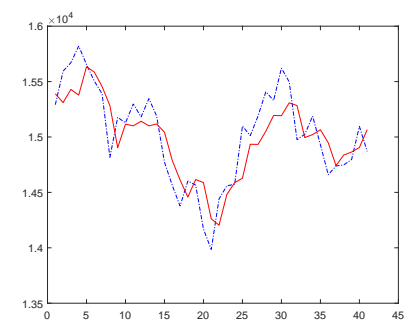

(c) 2000

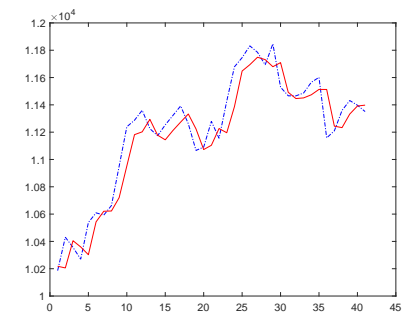

(d) 2001

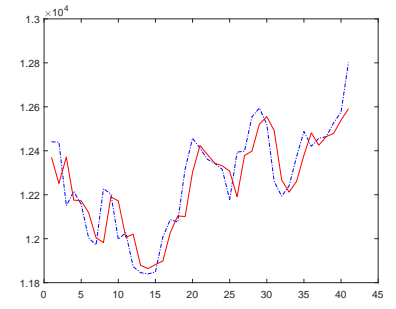

(f) 2003

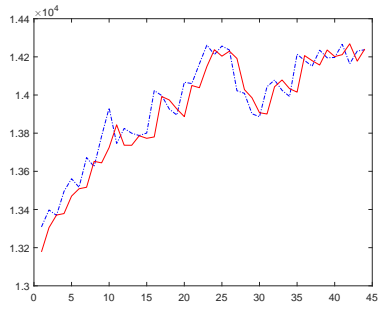

(g) 2004

Fig. 5. HSI forecasting results from 1997 to 2003

TABLE $\mathrm{V}$

A PERFORMANCE COMPARISON (RMSE) FOR FORECASTING HSI FROM 1998 TO 2003.

\begin{tabular}{lllllllll}
\hline Model & 1998 & 1999 & 2000 & 2001 & 2002 & 2003 & 2004 & Average \\
\hline AR(1) or ARIMA $(1,1,0)$ & 195.36 & 232.54 & 255.52 & 155.45 & 106.24 & 122.59 & 112.81 & 168.64 \\
AR(2) or ARIMA $(2,1,0)$ & 196.40 & 227.65 & 257.00 & 154.55 & 106.37 & 122.53 & 111.49 & 168.00 \\
FTSGA [98] (2013) & 202.66 & 217.09 & 246.12 & 163.33 & 104.28 & 129.00 & 101.29 & 166.25 \\
INFS+SVR [93] & 216.39 & 285.05 & 254.16 & 158.53 & 108.98 & 126.86 & 113.21 & 180.45 \\
Multiorder fuzzy [92] (2016) & 194.28 & 220.87 & 240.31 & 159.27 & 95.01 & 127.21 & 81.98 & 159.85 \\
INFS + ANFIS [93] (2016) & 201.18 & 235.68 & 249.28 & 157.97 & 105.6 & 124.15 & 103.28 & 168.16 \\
The proposed model $(\alpha=0.8)$ & 180.24 & 226.36 & 233.91 & 140.44 & 93.09 & 114.15 & 96.85 & 155.01 \\
\hline
\end{tabular}

TABLE VI

Performance improvement Degree (\%) FOR FOrecAsting HSI From 1998 to 2003.

\begin{tabular}{lllllllll}
\hline Models & 1998 & 1999 & 2000 & 2001 & 2002 & 2003 & 2004 & Average \\
\hline AR(1) or ARIMA $(1,1,0)$ & 7.74 & 2.66 & 8.46 & 9.66 & 12.38 & 6.88 & 14.15 & 8.08 \\
AR(2) or ARIMA (2,1,0) & 8.23 & 0.57 & 8.98 & 9.13 & 12.48 & 6.84 & 13.13 & 7.73 \\
FTSGA [98] (2013) & 11.06 & -4.27 & 4.96 & 14.01 & 10.73 & 11.51 & 4.38 & 6.76 \\
INFS+SVR [93] & 16.71 & 20.59 & 7.97 & 11.41 & 14.58 & 10.02 & 14.45 & 14.10 \\
Multiorder fuzzy [92] (2016) & 7.23 & -2.49 & 2.66 & 11.82 & 2.02 & 10.27 & -18.14 & 3.03 \\
INFS + ANFIS [93] (2016) & 10.41 & 3.95 & 6.17 & 11.10 & 11.85 & 8.05 & 6.23 & 7.82 \\
\hline
\end{tabular}

classic autoregression model.

Note that in the final step, IOWA is adopted to obtain the final prediction. In fact, this IOWA can be seen as a kind of aggregation framework $\vec{W}$. If we further adopt the maximal entropy method [78], the only parameter $\alpha$ should be well considered. TABLE VII and TABLE VIII make a comparison for the forecasting results with different selections of $\alpha$ or the degree of "orness". Note that we only consider $\alpha \geq 0.5$ because according to Equation (7), (20) and the related maximal entropy rules [78], parameter $\alpha$ characterizes a preference for specific time-frequency spaces with comparatively small errors in the past prediction process and Equation (20) indicates that we should prefer more these spaces with small accumulative errors, i.e., the $\alpha$ should be larger than 0.5. Particularly, if $\alpha=0.5$, the IOWA method becomes a simple mean calculation. From the above tables (TABLE VII and VIII) and figures (Figs. 6(a)\&6(b)), we can find that in the experiments, 0.8 is perhaps not the best choice and sometimes the simple mean can even do better. However, the differences among various $\alpha$ are not very large, and thus the proposed model can be said to be 


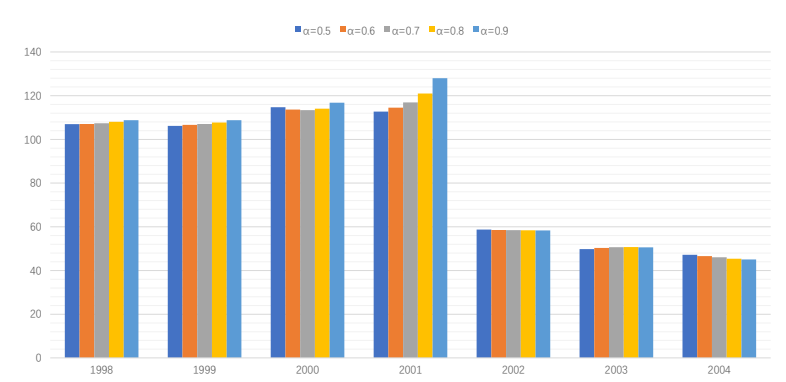

(a) A comparison of TAIEX forecasting results (RMSE) using different "orness"

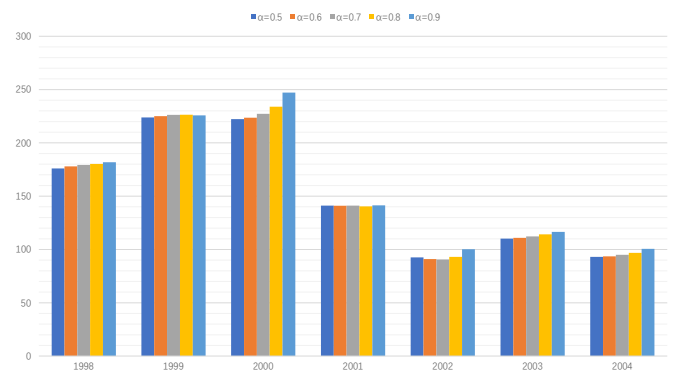

(b) A comparison of TAIEX forecasting results (RMSE) using different "orness"

Fig. 6. Forecasting result using different $\alpha$ or "orness".

stable to some extent, because the parameter does not have a drastic influence on the model's performance.

\section{CONCLUSION}

In this paper, a multiple time-frequency spaces fuzzy interval forecasting model is proposed for energy and finance forecasting modeling. First, the original time series is decomposed and reconstructed in different timefrequency spaces using the EEMD method. In this situation, we can consider decomposed components separately and value the specific ones which are easy to forecast more. Then, every subseries is transformed into a corresponding visibility graph by exploring the potential link relations in the times series. After the LRW link prediction method is adopted, the forecasting interval for each subseries can be obtained, which is used to avoid inaccurately direct forecasting, and better represent the uncertainty in the prediction process.

Inspired by the golden rule representation values, a novel rule-based representation function is proposed to select proper representation values for these forecasting intervals. Please note that in this rule-based way, we can adapt the proposed model to different application contexts by adopting different rules. Eventually, by means of the IOWA operator, the forecasting results in different time-frequency spaces will be aggregated, and then, the final prediction will be made. Two study cases with improved forecasting accuracy compared with welldeveloped traditional or recent time series forecasting models can illustrate the advantages of the proposed method in real energy and stock market time series. Besides, the proposed model has few parameters and is stable, because the IOWA parameter $\alpha$ has little influence on the final results.

The proposed model considers time series in a network view and then performs the forecasting by link prediction methods. Because the process of transforming the time series into the complex network is strictly irreversible, the forecasting results from the network nodes to values are seen as fuzzy intervals according to the weakly reversed visibility algorithm and the most recent time series real value. Our proposed method still has some limitations. First, it cannot handle multivariate
TABLE VII

TAIEX FORECASTING RESULTS (RMSE) WITH DIFFERENT ORNESS $(\alpha)$

\begin{tabular}{llllll}
\hline Year & $\alpha=0.5$ & $\alpha=0.6$ & $\alpha=0.7$ & $\alpha=0.8$ & $\alpha=0.9$ \\
\hline 1998 & 106.97 & 107.04 & 107.41 & 108.06 & 108.81 \\
1999 & 106.17 & 106.63 & 107.04 & 107.69 & 108.78 \\
2000 & 114.71 & 113.62 & 113.40 & 114.08 & 116.80 \\
2001 & 112.72 & 114.52 & 116.94 & 120.97 & 127.99 \\
2002 & 58.71 & 58.57 & 58.44 & 58.38 & 58.37 \\
2003 & 49.80 & 50.33 & 50.70 & 50.74 & 50.57 \\
2004 & 47.22 & 46.60 & 46.03 & 45.38 & 45.03 \\
\hline
\end{tabular}

TABLE VIII

HSI FORECASTING RESULTS (RMSE) WITH DIFFERENT ORNESS $(\alpha)$

\begin{tabular}{llllll}
\hline Year & $\alpha=0.5$ & $\alpha=0.6$ & $\alpha=0.7$ & $\alpha=0.8$ & $\alpha=0.9$ \\
\hline 1998 & 176.05 & 177.96 & 179.23 & 180.24 & 181.83 \\
1999 & 223.82 & 225.02 & 226.21 & 226.36 & 225.78 \\
2000 & 222.23 & 223.63 & 227.26 & 233.91 & 247.16 \\
2001 & 141.19 & 141.00 & 141.16 & 140.44 & 141.43 \\
2002 & 92.58 & 91.00 & 90.64 & 93.09 & 100.18 \\
2003 & 110.17 & 110.86 & 112.22 & 114.15 & 116.48 \\
2004 & 93.04 & 93.50 & 94.98 & 96.85 & 100.60 \\
\hline
\end{tabular}

time series because the visibility algorithm can transform only univariate time series into networks. Another limitation is the golden rules used to select representation values, which may require further improvement for suitable adaptation to the time series forecasting context. In addition, the recovery of interval time series from networks requires further consideration; in particular, further study of multivariate time series forecasting models from the network perspective will be necessary to improve the prediction accuracy in more complex situations. Moreover, the question of how to obtain more suitable fuzzy forecasting intervals deserve is worthy of further investigation.

\section{ACKNOWLEDGMENTS}

The authors greatly appreciate the reviewers' suggestions and the editor's encouragement. This research is supported by the Research Project of Education and Teaching Reform in Southwest University (No. 2019JY053), Fundamental Research Funds for the Central 
Universities (No. XDJK2019C085) and Chongqing Overseas Scholars Innovation Program (No. cx2018077).

\section{REFERENCES}

[1] M. S. Kıran, E. Özceylan, M. Gündüz, and T. Paksoy, “A novel hybrid approach based on particle swarm optimization and ant colony algorithm to forecast energy demand of Turkey," Energy Conversion and Management, vol. 53, no. 1, pp. 75-83, 2012.

[2] M. S. KiRan, E. ÖZceylan, M. GüNdüZ, and T. Paksoy, "Swarm intelligence approaches to estimate electricity energy demand in Turkey," Knowledge-Based Systems, vol. 36, pp. 93-103, 2012.

[3] P. Taylan, F. Yerlikaya-Özkurt, and G. Weber, "An approach to the mean shift outlier model by Tikhonov regularization and conic programming," Intell. Data Anal., vol. 18, no. 1, pp. 79-94, 2014.

[4] F. Yerlikaya-Özkurt, A. Askan, and G. Weber, "A hybrid computational method based on convex optimization for outlier problems: Application to earthquake ground motion prediction," Informatica, Lith. Acad. Sci., vol. 27, no. 4, pp. 893-910, 2016.

[5] F. Yerlikaya-Özkurt, C. Vardar-Acar, Y. Yolcu-Okur, and G. Weber, "Estimation of the Hurst parameter for fractional Brownian motion using the CMARS method," J. Computational Applied Mathematics, vol. 259, pp. 843-850, 2014.

[6] A. Özmen, Y. Yılmaz, and G.-W. Weber, "Natural gas consumption forecast with MARS and CMARS models for residential users," Energy Economics, vol. 70, pp. 357-381, 2018.

[7] Y. Yilmaz, R. Kurz, A. Özmen, and G.-W. Weber, "A new algorithm for scheduling condition-based maintenance of gas turbines," in ASME Turbo Expo 2015: Turbine Technical Conference and Exposition. American Society of Mechanical Engineers Digital Collection, 2015, doi: 10.1115/GT2015-43545.

[8] I. Livada, A. Synnefa, S. Haddad, R. Paolini, S. Garshasbi, G. Ulpiani, F. Fiorito, K. Vassilakopoulou, P. Osmond, and M. Santamouris, "Time series analysis of ambient air-temperature during the period 1970-2016 over Sydney, Australia," Science of The Total Environment, vol. 648, pp. 1627-1638, 2019.

[9] S. B. Tsai, Y.-C. Lee, and J.-J. Guo, "Using modified grey forecasting models to forecast the growth trends of green materials," Proceedings of the Institution of Mechanical Engineers, Part B: Journal of Engineering Manufacture, vol. 228, no. 6, pp. 931-940, 2014.

[10] S.-M. Chen and N.-Y. Chung, "Forecasting enrollments using high-order fuzzy time series and genetic algorithms," International Journal of Intelligent Systems, vol. 21, no. 5, pp. 485-501, 2006.

[11] A. G. Cherstvy, D. Vinod, E. Aghion, A. V. Chechkin, and R. Metzler, "Time averaging, ageing and delay analysis of financial time series," New Journal of Physics, vol. 19, no. 6, pp. 1-11, 2017, doi: 10.1088/1367-2630/aa7199.

[12] A. Rubio, J. D. Bermúdez, and E. Vercher, "Improving stock index forecasts by using a new weighted fuzzy-trend time series method," Expert Systems with Applications, vol. 76, pp. 12-20, 2017.

[13] F. Xiao, Z. Zhang, and J. Abawajy, "Workflow scheduling in distributed systems under fuzzy environment," Journal of Intelligent E Fuzzy Systems, vol. 37, no. 4, pp. 5323-5333, 2019.

[14] W.-c. Wang, K.-w. Chau, D.-m. Xu, and X.-Y. Chen, "Improving forecasting accuracy of annual runoff time series using ARIMA based on EEMD decomposition," Water Resources Management, vol. 29, no. 8, pp. 2655-2675, 2015.

[15] I. Gungor, B. Emiroğlu, A. ÇINAR, and M. Kıran, "Integration search strategies in tree seed algorithm for high dimensional function optimization," International Journal of Machine Learning and Cybernetics, 2019, doi: 10.1007/s13042-019-00970-1.

[16] A. Babalik, A. Ozkis, S. A. Uymaz, and M. S. Kiran, "A multi-objective artificial algae algorithm," Applied Soft Computing, vol. 68, pp. 377-395, 2018.

[17] S. Jafarian-Namin, A. Goli, M. Qolipour, A. Mostafaeipour, and A.-M. Golmohammadi, "Forecasting the wind power generation using Box-Jenkins and hybrid artificial intelligence: A case study," International Journal of Energy Sector Management, vol. 13, no. 4, pp. 1038-1062, 2019.

[18] A. Mostafaeipour, A. Goli, and M. Qolipour, "Prediction of air travel demand using a hybrid artificial neural network (ANN) with Bat and Firefly algorithms: A case study," The Journal of Supercomputing, vol. 74, no. 10, pp. 5461-5484, 2018.
[19] A. Goli, H. Khademi Zareh, R. Tavakkoli-Moghaddam, and A. Sadeghieh, "A comprehensive model of demand prediction based on hybrid artificial intelligence and metaheuristic algorithms: A case study in dairy industry," Journal of Industrial and Systems Engineering, vol. 11, no. 4, pp. 190-203, 2018.

[20] M. G. Frei and I. Osorio, "Intrinsic time-scale decomposition: time-frequency-energy analysis and real-time filtering of nonstationary signals," Proceedings of the Royal Society A: Mathematical, Physical and Engineering Sciences, vol. 463, no. 2078, pp. 321-342, 2006.

[21] Z. Wu and N. E. Huang, "Ensemble empirical mode decomposition: a noise-assisted data analysis method," Advances in Adaptive Data Analysis, vol. 1, no. 01, pp. 1-41, 2009.

[22] R. Zhang, B. Ashuri, and Y. Deng, "A novel method for forecasting time series based on fuzzy logic and visibility graph," Advances in Data Analysis and Classification, vol. 11, no. 4, pp. 759-783, 2017.

[23] F. Xiao, "EFMCDM: Evidential fuzzy multicriteria decision making based on belief entropy," IEEE Transactions on Fuzzy Systems, 2019, doi: 10.1109/TFUZZ.2019.2936368.

[24] - "A distance measure for intuitionistic fuzzy sets and its application to pattern classification problems," IEEE Transactions on Systems, Man, and Cybernetics: Systems, 2019, doi: 10.1109/TSMC.2019.2958635.

[25] E. Kropat, A. Özmen, G.-W. Weber, S. Meyer-Nieberg, and O. Defterli, "Fuzzy prediction strategies for gene-environment networks-Fuzzy regression analysis for two-modal regulatory systems," RAIRO-Operations Research, vol. 50, no. 2, pp. 413-435, 2016.

[26] L. Maciel, R. Ballini, and F. Gomide, "Evolving granular analytics for interval time series forecasting," Granular Computing, vol. 1, no. 4, pp. 213-224, 2016.

[27] _ "Evolving possibilistic fuzzy modeling for realized volatility forecasting with jumps," IEEE Transactions on Fuzzy Systems, vol. 25, no. 2, pp. 302-314, 2016.

[28] M. Figueiredo, R. Ballini, S. Soares, M. Andrade, and F. Gomide, "Learning algorithms for a class of neurofuzzy network and application," IEEE Transactions on Systems, Man, and Cybernetics, Part C (Applications and Reviews), vol. 34, no. 3, pp. 293-301, 2004.

[29] E. Kropat, R. A. Tikidji-Hamburyan, and G.-W. Weber, "Operations research in neuroscience," Annals of Operations Research, vol. 258, no. 1, pp. 1-4, Nov 2017, doi: 10.1007/s10479-017-2633-x.

[30] G. Weber, O. Defterli, S. Z. A. Gök, and E. Kropat, "Modeling, inference and optimization of regulatory networks based on time series data," European Journal of Operational Research, vol. 211, no. 1, pp. 1-14, 2011

[31] L. Lacasa, B. Luque, F. Ballesteros, J. Luque, and J. C. Nuno, "From time series to complex networks: The visibility graph," Proceedings of the National Academy of Sciences, vol. 105, no. 13, pp. 4972-4975, 2008.

[32] P. Xu, R. Zhang, and Y. Deng, "A novel visibility graph transformation of time series into weighted networks," Chaos, Solitons $\mathcal{E}$ Fractals, vol. 117, pp. 201-208, 2018.

[33] W. Jiang, B. Wei, J. Zhan, C. Xie, and D. Zhou, "A visibility graph power averaging aggregation operator: A methodology based on network analysis," Computers $\mathcal{E}$ Industrial Engineering, vol. 101, pp. 260-268, 2016

[34] L. Lü, L. Pan, T. Zhou, Y.-C. Zhang, and H. E. Stanley, "Toward link predictability of complex networks," Proceedings of the National Academy of Sciences, vol. 112, no. 8, pp. 2325-2330, 2015.

[35] V. Martínez, F. Berzal, and J.-C. Cubero, "A survey of link prediction in complex networks," ACM Computing Surveys (CSUR), vol. 49 , no. 4, p. 69,2017 , doi: $10.1145 / 3012704$

[36] S. Mao and Y. Fu, "Time series forecasting based on complex network analysis," IEEE Access, vol. 7, pp. 40 220-40 229, 2019.

[37] J. Arroyo, R. Espínola, and C. Maté, "Different approaches to forecast interval time series: a comparison in finance," Computational Economics, vol. 37, no. 2, pp. 169-191, 2011.

[38] L. Wang, X. Liu, and W. Pedrycz, "Effective intervals determined by information granules to improve forecasting in fuzzy time series," Expert Systems with Applications, vol. 40, no. 14, pp. 56735679, 2013.

[39] D. Leite, P. Costa Jr, and F. Gomide, "Evolving granular neural network for fuzzy time series forecasting," The 2012 International Joint Conference on Neural Networks, pp. 10-15, 2012, doi: 10.1109/IJCNN.2012.6252382.

[40] S. K. Das and S. K. Roy, "Effect of variable carbon emission in a multi-objective transportation-p-facility location problem under 
neutrosophic environment," Computers \& Industrial Engineering, vol. 132, pp. 311-324, 2019.

[41] S. K. Das, S. K. Roy, and G. W. Weber, "Heuristic approaches for solid transportation-p-facility location problem," Central European Journal of Operations Research, 2019, doi: 10.1007/s10100-019-006107.

[42] F. Xiao, "A new divergence measure for belief functions in D-S evidence theory for multisensor data fusion," Information Sciences, vol. 514, pp. 462-483, 2020.

[43] — - "Generalization of Dempster-Shafer theory: A complex mass function," Applied Intelligence, 2019, doi: 10.1007/s10489-01901617-y.

[44] S. K. Roy, G. Maity, and G.-W. Weber, "Multi-objective two-stage grey transportation problem using utility function with goals," Central European Journal of Operations Research, vol. 25, no. 2, pp. 417-439, 2017.

[45] S. K. Roy, M. Pervin, and G. W. Weber, "A two-warehouse probabilistic model with price discount on backorders under two levels of trade-credit policy," Journal of Industrial $\mathcal{E}$ Management Optimization, vol. 13, pp. 658-662, 2018, doi: 10.3934/jimo.2018167.

[46] S. K. Roy and A. Bhaumik, "Intelligent water management: A triangular type-2 intuitionistic fuzzy matrix games approach," Water Resources Management, vol. 32, no. 3, pp. 949-968, 2018.

[47] S. K. Roy, S. Midya, and G.-W. Weber, "Multi-objective multiitem fixed-charge solid transportation problem under twofold uncertainty," Neural Computing and Applications, vol. 31, no. 12, pp. 8593-8613, 2019, doi: 10.1007/s00521-019-04431-2.

[48] S. K. Roy and S. Midya, "Multi-objective fixed-charge solid transportation problem with product blending under intuitionistic fuzzy environment," Applied Intelligence, vol. 49, no. 10, pp. 35243538, 2019, doi: 10.1007/s10489-019-01466-9.

[49] G. Maity, S. K. Roy, and J. L. Verdegay, "Analyzing multimodal transportation problem and its application to artificial intelligence," Neural Computing and Applications, 2019, doi: 10.1007/s00521-019-04393-5.

[50] A. Bhaumik, S. K. Roy, and G. W. Weber, "Hesitant intervalvalued intuitionistic fuzzy-linguistic term set approach in prisoners' dilemma game theory using TOPSIS: a case study on humantrafficking," Central European Journal of Operations Research, 2019, doi: $10.1007 / \mathrm{s} 10100-019-00638-9$.

[51] R. R. Yager and D. P. Filev, "Induced ordered weighted averaging operators," IEEE Transactions on Systems, Man, and Cybernetics, Part B (Cybernetics), vol. 29, no. 2, pp. 141-150, 1999.

[52] W. Jiang and S. Wang, "An uncertainty measure for intervalvalued evidences." International Journal of Computers, Communications \& Control, vol. 12, no. 5, pp. 631-644, 2017.

[53] S.-M. Chen and Z.-C. Huang, "Multiattribute decision making based on interval-valued intuitionistic fuzzy values and linear programming methodology," Information Sciences, vol. 381, pp. 341-351, 2017.

[54] R. R. Yager and N. Alajlan, "Multicriteria decision-making with imprecise importance weights," IEEE Transactions on Fuzzy Systems, vol. 22, no. 4, pp. 882-891, 2014.

[55] H. Yang, Y. Deng, and J. Jones, "Network division method based on cellular growth and Physarum-inspired network adaptation," International Journal of Unconventional Computing, vol. 13, no. 6, pp. 477-491, 2018.

[56] D. Meng, Y. Li, S.-P. Zhu, G. Lv, J. Correia, and A. De Jesus, "An enhanced reliability index method and its application in reliability-based collaborative design and optimization," Mathematical Problems in Engineering, vol. 2019, 2019, doi: $10.1155 / 2019 / 4536906$.

[57] W. Deng and Y. Deng, "Entropic methodology for entanglement measures," Physica A: Statistical Mechanics and its Applications, vol. 512, pp. 693-697, 2018.

[58] X. Cao and Y. Deng, "A new geometric mean FMEA method based on information quality," IEEE Access, vol. 7, no. 1, pp. 95547-95554, 2019.

[59] D. Meng, S. Yang, Y. Zhang, and S.-P. Zhu, "Structural reliability analysis and uncertainties-based collaborative design and optimization of turbine blades using surrogate model," Fatigue $\mathcal{E}$ Fracture of Engineering Materials \& Structures, vol. 42, no. 6, pp. 1219-1227, 2019, doi: 10.1111/ffe.12906.

[60] W. Zhang and Y. Deng, "Combining conflicting evidence using the dematel method," Soft Computing, vol. 23, no. 17, pp. 82078216, 2019.
[61] H. Xu and Y. Deng, "Dependent evidence combination based on decision-making trial and evaluation laboratory method," International Journal of Intelligent Systems, vol. 34, no. 7, pp. 15551571, 2019.

[62] S. B. Tsai, M. F. Chien, Y. Xue, X. Li, Lei andJiang, Q. Chen, J. Zhou, and L. Wang, "Using the fuzzy dematel to determine environmental performance: A case of printed circuit board industry in Taiwan," Plos One, vol. 10, no. 6, pp. 1-18, 2015, doi: 10.1371/journal.pone.0129153.

[63] Z. He and W. Jiang, "An evidential Markov decision making model," Information Sciences, vol. 467, pp. 357-372, 2018.

[64] - "A new belief Markov chain model and its application in inventory prediction," International Journal of Production Research, vol. 56, no. 8, pp. 2800-2817, 2018.

[65] B. Wei and Y. Deng, "A cluster-growing dimension of complex networks: From the view of node closeness centrality," Physica A: Statistical Mechanics \& Its Applications, vol. 522, pp. 80 - 87, 2019.

[66] Y. Yang, R. N. Lichtenwalter, and N. V. Chawla, "Evaluating link prediction methods," Knowledge and Information Systems, vol. 45, no. 3, pp. 751-782, 2015.

[67] W. Liu and L. Lü, "Link prediction based on local random walk," EPL (Europhysics Letters), vol. 89, no. 5, p. 58007, 2010, doi: 10.1209/0295-5075/89/58007.

[68] M. Li, H. Xu, and Y. Deng, "Evidential decision tree based on belief entropy," Entropy, vol. 21, no. 9, p. 897, 2019.

[69] T. Zhou, L.-L. Jiang, R.-Q. Su, and Y.-C. Zhang, "Effect of initial configuration on network-based recommendation," EPL (Europhysics Letters), vol. 81, no. 5, p. 58004, 2008.

[70] D. Liben-Nowell and J. Kleinberg, "The link-prediction problem for social networks," Journal of the American Society for Information Science and Technology, vol. 58, no. 7, pp. 1019-1031, 2007.

[71] N. E. Huang, Z. Shen, S. R. Long, M. C. Wu, H. H. Shih, Q. Zheng, N.-C. Yen, C. C. Tung, and H. H. Liu, "The empirical mode decomposition and the Hilbert spectrum for nonlinear and nonstationary time series analysis," Proceedings of the Royal Society of London. Series A: Mathematical, Physical and Engineering Sciences, vol. 454, no. 1971, pp. 903-995, 1998.

[72] N. E. Huang, M.-L. C. Wu, S. R. Long, S. S. Shen, W. Qu, P. Gloersen, and K. L. Fan, "A confidence limit for the empirical mode decomposition and Hilbert spectral analysis," Proceedings of the Royal Society of London. Series A: Mathematical, Physical and Engineering Sciences, vol. 459, no. 2037, pp. 2317-2345, 2003.

[73] Y. Song and Y. Deng, "A new soft likelihood function based on power ordered weighted average operator," International Journal of Intelligent Systems, vol. 34, no. 11, pp. 2988-2999, 2019.

[74] R. R. Yager, "OWA aggregation of multi-criteria with mixed uncertain satisfactions," Information Sciences, vol. 417, pp. 88-95, 2017.

[75] R. R. Yager and N. Alajlan, "Probabilistically weighted OWA aggregation," IEEE Transactions on Fuzzy Systems, vol. 22, no. 1, pp. 46-56, 2014.

[76] R. R. Yager, "On ordered weighted averaging aggregation operators in multicriteria decisionmaking," IEEE Transactions on systems, Man, and Cybernetics, vol. 18, no. 1, pp. 183-190, 1988.

[77] —, "OWA aggregation with an uncertainty over the arguments," Information Fusion, vol. 52, pp. 206-212, 2019.

[78] R. Fullér and P. Majlender, "An analytic approach for obtaining maximal entropy OWA operator weights," Fuzzy Sets and Systems, vol. 124, no. 1, pp. 53-57, 2001.

[79] R. R. Yager, "Generalized Dempster-Shafer structures," IEEE Transactions on Fuzzy Systems, vol. 27, no. 3, pp. 428-435, 2019.

[80] Y. Dong, J. Zhang, Z. Li, Y. Hu, and Y. Deng, "Combination of evidential sensor reports with distance function and belief entropy in fault diagnosis," International Journal of Computers Communications \& Control, vol. 14, no. 3, pp. 329-343, 2019.

[81] Y. Song and Y. Deng, "A new method to measure the divergence in evidential sensor data fusion," International Journal of Distributed Sensor Networks, vol. 15, no. 4, 2019, doi: $10.1177 / 1550147719841295$.

[82] R. Sun and Y. Deng, "A new method to determine generalized basic probability assignment in the open world," IEEE Access, vol. 7, no. 1, pp. 52 827-52 835, 2019.

[83] M. Zhou, X.-B. Liu, J.-B. Yang, Y.-W. Chen, and J. Wu, "Evidential reasoning approach with multiple kinds of attributes and entropybased weight assignment," Knowledge-Based Systems, vol. 163, pp. 358-375, 2019. 
[84] M. Zhou, X.-B. Liu, Y.-W. Chen, and J.-B. Yang, "Evidential reasoning rule for MADM with both weights and reliabilities in group decision making," Knowledge-Based Systems, vol. 143, pp. 142-161, 2018.

[85] H. Liao, Z. Xu, and X.-J. Zeng, "Distance and similarity measures for hesitant fuzzy linguistic term sets and their application in multi-criteria decision making," Information Sciences, vol. 271, pp. 125-142, 2014.

[86] I. Dzitac, F. G. Filip, and M.-J. Manolescu, "Fuzzy logic is not fuzzy: World-renowned computer scientist Lotfi A. Zadeh," International Journal of Computers Communications \& Control, vol. 12, no. 6, pp. 748-789, 2017.

[87] B. Kang, P. Zhang, Z. Gao, G. Chhipi-Shrestha, K. Hewage, and R. Sadiq, "Environmental assessment under uncertainty using Dempster-Shafer theory and Z-numbers," Journal of Ambient Intelligence and Humanized Computing, 2019, doi: 10.1007/s12652-01901228-y.

[88] J. Zhao and Y. Deng, "Performer selection in Human Reliability analysis: D numbers approach," International Journal of Computers Communications \& Control, vol. 14, no. 3, pp. 437-452, 2019.

[89] T. Takagi and M. Sugeno, "Fuzzy identification of systems and its applications to modeling and control," IEEE Transactions on Systems, Man, and Cybernetics, no. 1, pp. 116-132, 1985.

[90] F. Liu and Y. Deng, "A fast algorithm for network forecasting time series," IEEE Access, vol. 7, no. 1, pp. 102554-102560, 2019.

[91] R. R. Yager, "Golden rule representative values for non-standard membership grades," 2016 IEEE 8th International Conference on Intelligent Systems (IS), pp. 2-7, 2016.

[92] F. Ye, L. Zhang, D. Zhang, H. Fujita, and Z. Gong, "A novel forecasting method based on multi-order fuzzy time series and technical analysis," Information Sciences, vol. 367, pp. 41-57, 2016.

[93] C.-H. Su and C.-H. Cheng, "A hybrid fuzzy time series model based on ANFIS and integrated nonlinear feature selection method for forecasting stock," Neurocomputing, vol. 205, pp. 264273, 2016.

[94] J. E. Nash and J. V. Sutcliffe, "River flow forecasting through conceptual models part I-A discussion of principles," Journal of Hydrology, vol. 10, no. 3, pp. 282-290, 1970.

[95] Q. Zhang, B.-D. Wang, B. He, Y. Peng, and M.-L. Ren, "Singular spectrum analysis and ARIMA hybrid model for annual runoff forecasting," Water Resources Management, vol. 25, no. 11, pp. 2683-2703, 2011

[96] G. E. Box, G. M. Jenkins, G. C. Reinsel, and G. M. Ljung, Time Series Analysis: Forecasting and Control. John Wiley \& Sons, 2015.

[97] J. Sullivan and W. H. Woodall, "A comparison of fuzzy forecasting and Markov modeling," Fuzzy Sets and Systems, vol. 64, no. 3, pp. 279-293, 1994.

[98] Q. Cai, D. Zhang, B. Wu, and S. C. Leung, "A novel stock forecasting model based on fuzzy time series and genetic algorithm," Procedia Computer Science, vol. 18, pp. 1155-1162, 2013. 